\title{
INFLUÊNCIA DAS CARACTERÍSTICAS FÍSICAS NA DETERMINAÇÃO DE PARÂMETROS HIDRÁULICOS DE RESÍDUOS SÓLIDOS URBANOS
}

\author{
André Geraldo Cornélio RIBEIRO 1; Rodrigo César de Vasconcelos dos SANTOS ${ }^{2}$; Carson \\ dos Santos NEVES ${ }^{3}$; Fátima Resende Luiz FIA ${ }^{4}$; Rosângela Francisca de Paula Vitor \\ MARQUES ${ }^{5}$
}

\footnotetext{
${ }^{1}$ Engenheiro Civil. Doutor em Engenharia Civil. Professor Adjunto do Departamento de Engenharia, setor de Engenharia Ambiental e Sanitária da Universidade Federal de Lavras. E-mail: andreribeiro@deg.ufla.br.

${ }^{2}$ Engenheiro Ambiental e Sanitarista (UFLA). Doutorando em Recursos Hídricos Departamento de Engenharia, Universidade Federal de Pelotas. E-mail: drigovasc@hotmail.com.

3 Engenheiro Ambiental e Sanitarista, Departamento de Engenharia, Universidade Federal de Lavras. E-mail: carsonba@hotmail.com.

${ }^{4}$ Engenheira Agrícola. Doutora em Engenharia Agrícola. Professora Adjunta do Departamento de Engenharia, setor de Engenharia Ambiental e Sanitária da Universidade Federal de Lavras. E-mail: fatimarlf@deg.ufla.br.

${ }^{5}$ Engenheira Florestal. Doutora em Recursos Hídricos em Sistemas Agrícolas (UFLA). Professora tempo integral - Mestrado em Sustentabilidade em Recursos Hídricos (UNINCOR) E-mail: roeflorestal@hotmail.com.
}

Recebido em: 10/09/2017 - Aprovado em: 02/11/2017 - Disponibilizado em: 30/12/2017

Resumo: Nos projetos de aterros sanitários brasileiros são utilizados parâmetros de resíduos advindos de países europeus e da América do Norte. Entretanto, os resíduos possuem características diferenciadas conforme a renda da população, hábitos culturais, clima, entre outros. A fim de melhorar os procedimentos relativos à disposição dos resíduos sólidos urbanos (RSU) em aterros sanitários, faz-se necessário a determinação de parâmetros de acordo com a realidade brasileira. Neste contexto, objetivou-se neste trabalho avaliar a influência da composição gravimétrica e do peso específico $(\gamma)$ no valor da condutividade hidráulica $(k)$ e da capacidade de campo $(\theta)$ de resíduos sólidos urbanos (RSU). Amostras de RSU foram coletadas na usina de triagem e compostagem (UTC) da cidade de Nazareno e também de restaurantes da cidade de Lavras, no estado de Minas Gerais, no Brasil. O artigo apresenta detalhes do equipamento desenvolvido, bem como da metodologia usada e os resultados encontrados. As conclusões apontam que o equipamento mostrou-se adequado para os procedimentos adotados. Além disso, pode-se concluir que houve uma diminuição da condutividade hidráulica e um aumento da capacidade de campo para as amostras de RSU com maior peso específico. Porém, não foram observadas variações significativas desses parâmetros em relação à composição gravimétrica, provavelmente devido ao tipo e qualidade dos materiais utilizados.

Palavras-chave: Condutividade hidráulica. Capacidade de campo. Aterro sanitário.

\section{INFLUENCE OF PHYSICAL CHARACTERISTICS ON THE HYDRAULIC PARAMETERS DETERMINATION OF URBAN SOLID RESIDUES}

\begin{abstract}
In Brazilian landfill projects waste parameters from European and North American countries are used. However, the residues have different characteristics according to the income of the population, cultural habits, climate, among others. In order to improve the procedures related to the disposal of municipal solid waste (MSW) in landfills, it is necessary to determine parameters according to the Brazilian reality. In this context, the objective of this study was to evaluate the influence of gravimetric composition and specific gravity on the hydraulic conductivity value and the field capacity of municipal solid waste. Samples of MSW were collected on the sorting and composting plant in Nazareno and also from restaurants in Lavras, both cities in Minas Gerais, Brazil. This paper brings up details from the developed equipment, also from the methodology used and the obtained results. Conclusions points out that the equipment suits the employed procedures. Besides, it can be concluded that there was a decrease on the hydraulic conductivity values and an increase on the field capacity for the MSW samples with bigger specific weight. However, no significant variations were observed for these parameters in relation with the gravimetric compositions, probably due to the type and quality of the utilized materials.
\end{abstract}

Keywords: Hydraulic conductivity. Field capacity. Landfill.

\section{INTRODUÇÃO}

Entre os desafios ambientais do país, a grande geração de resíduos sólidos urbanos (RSU) está entre os mais consideráveis e representativos. Problemas significativos foram observados, especialmente na última década, no tocante à diversificação e ao aumento no descarte de materiais produzidos pelas indústrias para suprir a demanda da população por bens manufaturados. Esse cenário aliado à má 
gestão praticada pelo poder público tem como consequência o surgimento de áreas clandestinas de deposição de resíduos, ocasionando a poluição do solo, da água e do ar, além da proliferação de vários vetores de doenças.

Desta forma, o esforço para encontrar soluções técnicas viáveis e ambientalmente corretas para disposição dos resíduos sólidos estudando as características desse material, é tema de vários trabalhos dos mais diversos pesquisadores como Ribeiro et al. (2013), Durmusoglu et al. (2006), Dixon; Jones (2005), Boscov; Abreu (2000), entre outros.

No intuito de contribuir com a solução dessa problemática relacionada aos RSU, instituiu-se a Política Nacional de Resíduos Sólidos (PNRS), por meio da Lei Federal $n^{\circ}$ $12.305 / 2010$, tem diretrizes na gestão integrada e no gerenciamento dos resíduos sólidos e, ainda, preconiza a disposição final dos rejeitos em aterros sanitários, proibindo, a partir de agosto de 2014, a disposição de resíduos por parte dos municípios em vazadouros a céu aberto (“lixões"), substituindo-os pela implantação de aterros sanitários. No entanto, o projeto de lei 2289/2015 em tramitação desde julho de 2015, propõe a prorrogação do prazo para os municípios erradicarem os lixões, adequarem o gerenciamento dos RSU e adotarem disposição dos rejeitos em aterros sanitários, com prazos escalonados que variam entre os anos de 2018 a 2021, de acordo com o porte do município.

Para o dimensionamento de aterros sanitários torna-se necessário conhecer algumas propriedades hidráulicas dos resíduos (Carvalho, 2002), de modo particular, a condutividade hidráulica $(k)$ e capacidade de campo ( $\theta)$ (Rocha, 2008).

A condutividade hidráulica é a propriedade hidráulica de um meio poroso que quantifica a facilidade de um líquido fluir por seus espaços vazios existentes entre suas partículas com maior ou menor velocidade (SILVEIRA, 2014). De acordo com Borgatto (2006), a condutividade hidráulica de RSU é um importante dado de projeto e operação de aterros sanitários, principalmente no dimensionamento do sistema de drenagem e tratamento de percolado e nos acontecimentos de problemas com estabilidade e migração não controlada de líquido percolado.

A capacidade de campo é a quantidade máxima de água retida por um meio poroso sem gerar percolação descendente, após o escoamento do seu excesso sob a ação da gravidade. Assim os líquidos percolados são produzidos quando o teor de umidade na massa do resíduo excede a capacidade de campo (EL-FADEL et al., 2002). Esta pode ser expressa em termos volumétricos ou gravimétricos. Alguns autores revelam que a capacidade de campo dos resíduos sólidos varia em torno de 22,7 a $51,4 \%$, 
dependendo das características dos resíduos ( Yuen et al (2001); Carvalho (2002); Lins (2003); Barros (2004); Carvalho (2006), Calle (2007).

Para Lins e Jucá (2005), a capacidade de campo dos resíduos em um aterro sanitário, muda rapidamente durante os primeiros dias de operação, por causa da circulação de veículos sobre os mesmos e pela aplicação da camada de cobertura. Os mesmos autores ainda relatam que o valor da capacidade de campo diminui com a idade dos resíduos, sendo justificada pela mineralização da matéria orgânica e pelo aumento do peso específico da massa de resíduos e a consequente redução de poros.

Esses parâmetros, Condutividade hidráulica e capacidade de campo, podem variar de acordo com algumas características físicas dos RSU, como: peso específico $(\gamma)$, idade dos resíduos, bem como sua composição gravimétrica, uma vez que trata-se de um material extremamente heterogêneo, cujo maior percentual em massa é de matéria orgânica (MO).

Segundo Carvalho (2006) e Ribeiro (2007), nos projetos de aterros sanitários

\section{MATERIAL E MÉTODOS}

Os resíduos sólidos urbanos utilizados no experimento foram provenientes da Usina de Triagem e brasileiros são utilizados parâmetros de resíduos advindos de países europeus e da América do Norte. Entretanto, sabe-se que os resíduos não são homogêneos e com propriedades fixas, sendo que as suas características são diferenciadas conforme a renda da população, hábitos culturais, clima, entre outros. Logo, faz-se necessário a determinação de parâmetros relacionados com a realidade dos nossos resíduos sólidos, a fim de melhorar os procedimentos relativos à disposição dos RSU em aterros sanitários.

Desta forma, entende-se que o estudo acerca da condutividade hidráulica e da capacidade de campo dos RSU é de suma importância para um dimensionamento adequado de aterros sanitários, evitando assim problemas sérios de contaminação do meio ambiente.

Diante deste contexto, o objetivo do presente trabalho foi avaliar a influência da composição gravimétrica e do peso específico no valor da condutividade hidráulica e da capacidade de campo de resíduos sólidos urbanos.

Compostagem (UTC) do município de Nazareno-MG e parte da matéria orgânica proveniente de um restaurante do município de Lavras-MG. Esses resíduos foram coletados separadamente em: papel, 
plástico, papelão, metais, rejeito e matéria orgânica. A matéria orgânica utilizada no presente é mais diversificada (restos de comida, de frutas, galhos e folhas, entre outros) devido aos locais de onde foram coletados.

Sendo os mesmos transportados para o Campo Experimental de Tratamento de Resíduos Sólidos (CETRES) da Universidade Federal de Lavras.

Para a realização dos ensaios, foram montadas três amostras de resíduos para o peso específico de $5 \mathrm{kN} \mathrm{m}^{-3}$, e três amostras de resíduos para o peso específico de $3 \mathrm{kN}$ $\mathrm{m}^{-3}$. As amostras para cada peso específico foram montadas com composições gravimétricas diferentes $(20,40$ e $60 \%$ de $\mathrm{MO})$, conforme especificado por Franco (2012) que estudou a composição dos resíduos sólidos domiciliares da região Sul de Minas Gerais, para o período do verão. Justifica-se a escolha dessas porcentagem de MO pelo seguinte fato: $20 \%$ de MO por ser o valor permitido a ser aterrado segundo a legislação vigente; $60 \%$ de MO seguindo a composição gravimétrica média brasileira e $40 \%$ de MO como valor intermediário.
Embora o experimento tenha ocorrido entre os meses de abril a dezembro de 2015, a escolha do período de verão foi para dar continuidade ao trabalho iniciado por Triginelli (2015), sendo que a referida autora utilizou esse período para suas análises, e também para manter um padrão de comparação entre os pesos específicos utilizados.

Para a montagem das amostras foi realizado a caracterização gravimétrica dos resíduos, sendo que os mesmos foram separados e agrupados em categorias:

1. Papelão: papelão e longa vida;

2. Papel: papel fino;

3. Plástico: plástico filme, plástico rígido, vidro branco;

4. Metal: metais ferrosos;

5. Rejeito: banheiro, entulho, eletrônico, mistura de materiais, tecidos e outros.

Para a determinação da condutividade hidráulica e da capacidade de campo foi utilizado um permeâmetro de carga constante e de grandes dimensões, desenvolvido por Triginelli (2015), baseado em Ribeiro et al (2013), conforme ilustrado na Figura 1. 
Figura 1 - Conjunto permeâmetro, reservatório e painel piezométrico utilizado para determinação da condutividade hidráulica e capacidade de campo dos RSU.

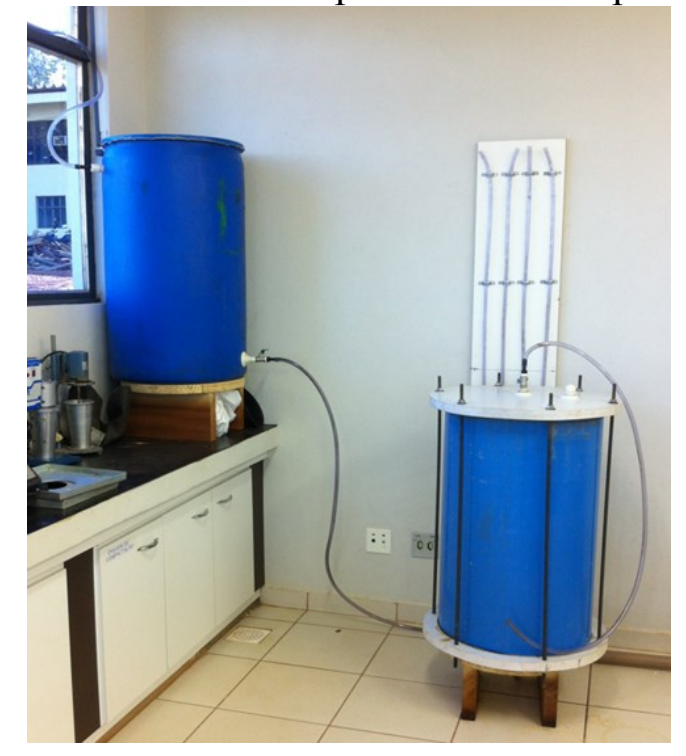

O permeâmetro de grandes dimensões utilizado, consiste em uma peça cilíndrica de PVC rígido, com $50 \mathrm{~cm}$ de diâmetro interno, $85 \mathrm{~cm}$ de altura, paredes de 2,5 cm de espessura, e tampas, também em PVC, com $65 \mathrm{~cm}$ de diâmetro e $3 \mathrm{~cm}$ de espessura, com aberturas para conexão de flanges com registros para alimentação e saída de água no permeâmetro. Na tampa superior há um orifício que auxilia na expulsão do ar durante a saturação da amostra de resíduos.

O permeâmetro foi alimentado de forma ascendente por um reservatório de 200 litros que funciona como um frasco de Mariotte, permitindo que os ensaios fossem realizados com carga constante. O mesmo também foi acoplado a um painel piezométrico através de quatro registros espaçados entre si de $15 \mathrm{~cm}$ ao longo da sua altura. Os registros conectados por meio de mangueiras ao painel possibilitou a leitura das cargas piezométricas em diferentes pontos da amostra.

Para que a pressão a ser medida nos ensaios fosse do centro da amostra, foram colocadas no interior da peça cilíndrica, conectadas aos registros, mangueiras com aproximadamente $15 \mathrm{~cm}$ de comprimento e $2,5 \mathrm{~cm}$ de diâmetro com furos ao longo do seu comprimento.

\section{Determinação da condutividade hidráulica}

Para a determinação da condutividade hidráulica utilizou-se a metodologia descrita por Triginelli (2015), baseada em Ribeiro et al (2013), onde os resíduos foram pesados, misturados na devida proporção, colocados no 
permeâmetro e posteriormente compactados com um soquete pesando aproximadamente $20 \mathrm{~kg}$, em camadas de 10 $\mathrm{cm}$ (Figuras 2a e b), de maneira a garantir um controle de compactação para que as amostras atingissem os pesos específicos de 3 e $5 \mathrm{kN} \mathrm{m}^{-3}$.

Figura 2 - Resíduos misturados na devida proporção por camada (A). Resíduos colocados no permeâmetro e compactados em camadas (B).
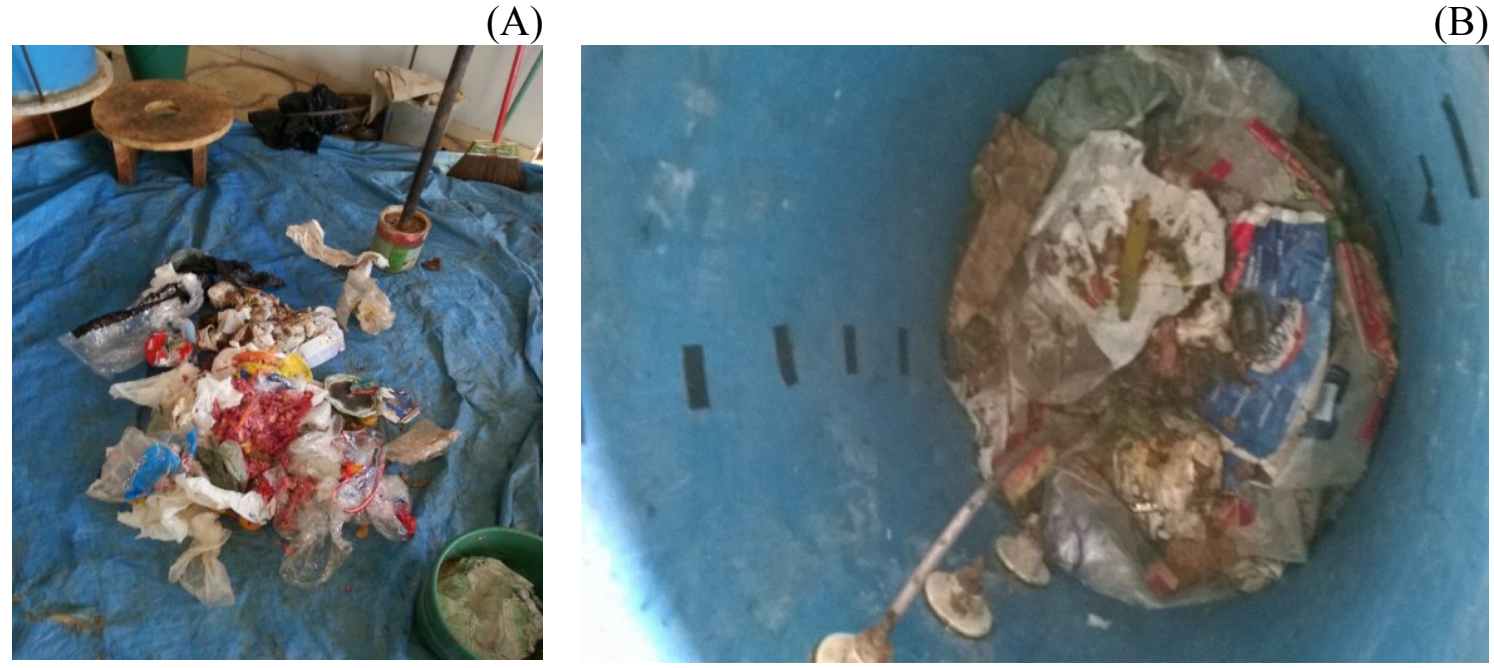

Antes e após o enchimento do permeâmetro foi colocada uma camada de 5 cm de brita $\mathrm{N}^{\circ} 1$ para evitar o entupimento pelos resíduos da entrada e saída de água e também para facilitar a drenagem.

Para o completo preenchimento do permeâmetro foi necessária uma massa total de $75 \mathrm{~kg}$ de resíduos para atingir o peso específico de $5 \mathrm{kN} \mathrm{m}^{-3}$, e uma massa de 45 $\mathrm{kg}$ de resíduos para atingir o peso específico de $3 \mathrm{kN} \mathrm{m}^{-3}$.

Após a realização da compactação, o permeâmetro foi fechado e devidamente vedado. Em seguida, iniciou-se o procedimento de saturação da amostra, onde o registro que alimenta o reservatório foi fechado e outro registro, situado na parte inferior do mesmo foi aberto para que se iniciasse a entrada de água no permeâmetro. Durante o processo de saturação, o orifício na tampa superior do permeâmetro, bem como o registro para saída de água foram abertos para auxiliar na expulsão do ar existente nos espaços vazios dos resíduos.

A amostra de resíduos foi considerada saturada quando, fechados os registros de entrada e saída de água do permeâmetro, as cargas piezométricas dos quatro piezômetros no painel apresentavam-se estabilizadas e niveladas na altura do permeâmetro.

Após confirmada a saturação, os registros foram novamente abertos e procedeu-se com a leitura dos níveis d'água 
nos piezômetros, na qual foram realizadas sete leituras para cada amostra compactada, determinando assim o gradiente hidráulico (i). Para obter um valor único, foi calculada a média dos gradientes hidráulicos (i) entre os quatro piezômetros instalados no permeâmetro. Porém, no ensaio com a amostra contendo $60 \%$ de $\mathrm{MO}$ e compactada a um peso específico de $3 \mathrm{kN}$ $\mathrm{m}^{-3}$, o registro do quarto piezômetro entupiu, sendo portanto, desconsiderado para o cálculo do gradiente hidráulico deste ensaio.

Para cada ensaio no permeâmetro, a vazão (Q) que passou pela amostra de RSU foi calculada com base no volume de água (V) coletado num intervalo de tempo de $\operatorname{coleta}(\Delta \mathrm{t})$.

Conforme sugerido por Ribeiro et al (2013), para dar confiabilidade aos dados levantados, pois se trata de uma massa extremamente heterogênea, utilizou-se o critério do "Intervalo de Confiança" (IC) (Equação 1), após o cálculo da média e desvio padrão do conjunto de valores de gradientes hidráulicos.

$$
\text { IC }(\mathrm{u})=\text { média } \pm \mathrm{t} * \frac{\text { desvpad }}{\sqrt{\mathrm{n}}}
$$

Em que:

$\mathrm{IC}=$ intervalo de confiança, média $=$ média dos valores de gradiente hidráulico, $\mathrm{n}=$ número de informações, $\mathrm{t}=$ distribuição de Student para o nível de significância $\alpha$ e n-
1 graus de liberdade desvpad = desviopadrão da média calculada.

No presente trabalho, a variável analisada foi o gradiente hidráulico, sendo “n” igual a 7, que corresponde ao número de leituras realizadas. $\mathrm{O}$ valor de " $\mathrm{t}$ " adotado foi de $1 \%$, significando que se tem $99 \%$ de confiança que o valor do gradiente hidráulico se encontra dentro dos limites estipulados.

Foram descartados os valores dos gradientes hidráulicos que se apresentaram fora do intervalo de confiança, calculandose novamente a média dos valores dos gradientes hidráulicos, utilizando-se apenas valores dentro do intervalo calculado.

Logo, calculou-se a condutividade hidráulica por meio da Equação (2).

$$
\mathrm{K}=\frac{\mathrm{Q}}{\mathrm{i} * \mathrm{~A}}
$$

Em que:

$\mathrm{Q}=$ vazão $\left(\mathrm{m}^{3} \mathrm{~s}^{-1}\right)$ média das 7 leituras realizadas, $\mathrm{i}=$ gradiente hidráulico médio $\left(\mathrm{m} \mathrm{m}^{-1}\right)$ e $\mathrm{A}=$ área da seção transversal ao escoamento $\left(\mathrm{m}^{2}\right)$.

\section{Determinação da capacidade de campo}

Ao final de cada ensaio de condutividade hidráulica, foram fechados os registros superior e inferior do permeâmetro e desconectado a mangueira que ligava o reservatório ao permeâmetro. Em seguida, abriram-se os registros 
superior e inferior coletando, em um recipiente graduado, o percolado que drenava pela saída inferior do permeâmetro. Durante o ensaio de capacidade de campo, os registros do painel piezométrico mantiveram-se fechados.

Como no início o volume de percolado produzido foi grande, media-se o volume em curtos intervalos de tempo. Com o passar das horas a vazão diminuiu e então fez a coleta diariamente. De acordo com Carvalho (2002, 2006), a coleta foi interrompida assim que a vazão atingisse um valor menor ou igual a $0,01 \mathrm{~cm}^{3} \mathrm{~s}^{-1}, \mathrm{o}$ que levou em média a duração de 2 dias para cada ensaio

Ao final de cada ensaio, abria-se o permeâmetro e retiravam-se amostras de resíduos com aproximadamente $2 \mathrm{~kg}$ do topo, meio e base para determinar a umidade gravimétrica correspondente à capacidade de campo.

A capacidade de campo foi determinada com base na Equação 3:

$$
\theta=\frac{\gamma_{\mathrm{d}} * \mathrm{~W}_{\theta}}{\gamma_{\mathrm{w}}}
$$

Sendo:

O teor de água (W) foi obtido em base úmida utilizando a Equação 5:

$\mathrm{W}(\%)=\frac{\mathrm{M} 1-\mathrm{M} 2}{\mathrm{M} 1} * 100$

Em que: $\theta=$ capacidade de campo $(\%)$;

$\gamma_{\mathrm{d}}=$ peso específico seco $\quad\left(\mathrm{kN} \mathrm{m}^{-3}\right), \mathrm{W}_{\theta}$

$=$ teor de água ou umidade gravimétrica correspondente à capacidade de campo $(\%)$; $\gamma_{\mathrm{w}}=$ peso específico da água $\left(\mathrm{kN} \mathrm{m}^{-3}\right)$.

O peso específico seco foi determinado por meio da Equação 4:

$$
\gamma_{\mathrm{d}}=\frac{\gamma_{\mathrm{t}}}{1+\mathrm{W}_{\mathrm{i}}}
$$

Sendo:

$\mathrm{W}_{\mathrm{i}}=$ teor de água inicial (\%); $\gamma_{\mathrm{t}}=$ peso específico total $\left(\mathrm{kN} \mathrm{m}^{-3}\right)$.

\section{Determinação do teor de água (umidade)}

Os teores de água ou umidades gravimétricas iniciais e os correspondentes à capacidade de campo dos resíduos foram determinados a partir de amostras com aproximadamente $3,464 \mathrm{~kg}$ de massa bruta úmida (MBU) (2 $\mathrm{kg}$ de resíduos e 1,464 kg da tara da bandeja).

Para determinação da massa bruta seca (MBS), as amostras foram levadas para uma estufa de secagem com ventilação, na temperatura de $60{ }^{\circ} \mathrm{C}$, onde ficaram até que a massa se tornasse constante, o que levou em média 15 dias para cada ensaio.

$$
\begin{aligned}
& \text { M1 }=\text { massa bruta úmida }(\mathrm{g}) \\
& \mathrm{M} 2=\text { massa bruta seca }(\mathrm{g}) .
\end{aligned}
$$

Os dados apresentados foram obtidos a partir da média aritmética de três determinações. 
RESULTADOS E DISCUSSÃO

\section{Composição gravimétrica das amostras de RSU}

Nas Tabelas 1 e 2, são apresentadas as composições gravimétricas das amostras de resíduos utilizadas para os ensaios com o peso específico de $5 \mathrm{kN} \mathrm{m}^{-3}$ e $3 \mathrm{kN} \mathrm{m}^{-3}$, respectivamente.

Tabela 1 - Composição gravimétrica da amostra de RSU para os ensaios com 20\%, 40\%, $60 \%$ de $\mathrm{MO}$ e peso específico de $5 \mathrm{kN} \mathrm{m}^{-3}$.

\begin{tabular}{lcccccc}
\hline & $\%$ & Massa $(\mathrm{Kg})$ & $\%$ & Massa $(\mathrm{Kg})$ & $\%$ & Massa $(\mathrm{Kg})$ \\
\hline MO & 20 & 15 & 40 & 30 & 60 & 45 \\
Plástico & 16 & 12 & 12 & 9 & 8 & 6 \\
Papel & 13 & 9,8 & 9 & 6,8 & 5 & 3,8 \\
Papelão & 13 & 9,8 & 9 & 6,8 & 5 & 3,8 \\
Metal & 10 & 7,5 & 6 & 4,5 & 2 & 1,5 \\
Rejeito & 28 & 21 & 24 & 18 & 20 & 15 \\
\hline \multicolumn{1}{c}{ Total } & 100 & 75,1 & 100 & 75,1 & 100 & 75,1 \\
\hline
\end{tabular}

Tabela 2 - Composição gravimétrica da amostra de RSU para os ensaios com 20\%, 40\%, $60 \%$ de $\mathrm{MO}$ e peso específico de $3 \mathrm{kN} \mathrm{m}^{-3}$.

\begin{tabular}{lcccccc}
\hline & $\%$ & Massa $(\mathrm{Kg})$ & $\%$ & Massa $(\mathrm{Kg})$ & $\%$ & Massa $(\mathrm{Kg})$ \\
\hline MO & 20 & 9 & 40 & 18 & 60 & 27 \\
Plástico & 16 & 7,2 & 12 & 5,4 & 8 & 3,6 \\
Papel & 13 & 5,9 & 9 & 4,1 & 5 & 2,3 \\
Papelão & 13 & 5,9 & 9 & 4,1 & 5 & 2,3 \\
Metal & 10 & 4,5 & 6 & 2,7 & 2 & 0,9 \\
Rejeito & 28 & 12,6 & 24 & 10,8 & 20 & 9 \\
\hline \multicolumn{1}{c}{ Total } & 100 & 45 & 100 & 45 & 100 & 45 \\
\hline
\end{tabular}

Conforme é possível observar nas Tabelas 1 e 2, partindo-se do ensaio com $60 \%$ de matéria orgânica, os $20 \%$ de matéria orgânica reduzidos em cada um dos outros dois ensaios foram redistribuídos igualmente entre os outros materiais, formando assim três amostras com composições gravimétricas diferentes para cada peso específico.

\section{Condutividade hidráulica}

Os valores encontrados para a condutividade hidráulica dos RSU para os ensaios com 3 e $5 \mathrm{kN} \mathrm{m}^{-3}$ são apresentados na Tabela 3. 
Tabela 3 - Valores encontrados de condutividade hidráulica em função do peso específico e composição dos resíduos.

\begin{tabular}{ccc}
\hline Peso específico & \% MO & $\mathbf{k ~ ( \mathbf { m ~ s } ^ { - 1 } )}$ \\
\hline \multirow{2}{*}{$5 \mathrm{kN} \mathrm{m}^{-3}$} & 20 & $3,57 \times 10^{-5}$ \\
& 40 & $2,53 \times 10^{-5}$ \\
& 60 & $7,52 \times 10^{-5}$ \\
\hline \multirow{2}{*}{$3 \mathrm{kN} \mathrm{m}^{-3}$} & 20 & $4,33 \times 10^{-4}$ \\
& 40 & $4,10 \times 10^{-4}$ \\
& 60 & $2,93 \times 10^{-3}$ \\
\hline
\end{tabular}

Observa-se na Tabela 3 que os RSU compactados com peso específico de $5 \mathrm{kN}$ $\mathrm{m}^{-3}$ apresentaram uma menor condutividade hidráulica do que os resíduos compactados com $3 \mathrm{kN} \mathrm{m}^{-3}$, ou seja, quanto maior o peso específico, menor a condutividade hidráulica, o que também foi verificado por diversos autores, tais como Machado et al. (2010), Borgatto (2010), Lemare Neto (2004), Rocha (2008), Ribeiro et al (2013) e Mortatti (2013). Tal fato ocorre devido à diminuição dos espaços vazios entre os resíduos compactados, diminuindo assim o fluxo de água na massa de resíduos. Outra explicação condizente é que quanto maior o peso específico, ou seja, maior a compactação dos resíduos, maior é a quantidade de massa de RSU em um mesmo volume, o que provoca maior resistência à percolação do líquido.

Segundo Mortatti (2013), resíduos com maior porcentagem de plásticos apresentam maior condutividade hidráulica, o que foi contrário ao encontrado neste trabalho. Possivelmente, os resíduos plásticos do referido autor estavam em tamanhos menores em comparação aos resíduos utilizados no presente trabalho.

Em relação à variação da condutividade hidráulica em função da composição gravimétrica dos resíduos, percebe-se que houve um decréscimo dos valores de $k$ do ensaio de $20 \%$ de MO para o de $40 \%$ de MO. No entanto, ocorreu um aumento da condutividade hidráulica nos ensaios com $60 \%$ de MO. De um modo geral, verifica-se que quanto maior a quantidade de matéria orgânica, menor foi o volume ocupado no permeâmetro pelos outros componentes da composição, facilitando assim a passagem do percolado.

Lemare Neto (2004) relata que grandes quantidades de matéria orgânica destinadas para os aterros sanitários brasileiros causam valores baixos de condutividade hidráulica. Já Machado et al (2010) afirmam que aterros com grande quantidade de plásticos tendem a ter menor condutividade hidráulica, pois estes causam grande obstrução do fluxo de percolado.

Considerando que no presente trabalho o peso específico e a composição 
gravimétrica foram controlados, pode-se afirmar que quanto maior a quantidade de matéria orgânica, menor a quantidade de plásticos compactados no permeâmetro. Assim segundo o que foi relatado por Machado et al (2010), a tendência é que os ensaios com maior quantidade de $\mathrm{MO}$, e consequentemente menor quantidade de plásticos, apresentem maior condutividade hidráulica, conforme observado.

Triginelli (2015) utilizando o mesmo permeâmetro do presente trabalho encontrou uma condutividade hidráulica igual a $1,57 \times 10^{-4} \mathrm{~m} \mathrm{~s}^{-1}$ para o ensaio com $60 \%$ de $\mathrm{MO}$ e $1,94 \times 10^{-5} \mathrm{~m} \mathrm{~s}^{-1}$ para o ensaio com $40 \%$ de $\mathrm{MO}$, ambos com peso específico de $5 \mathrm{kN} \mathrm{m}^{-3}$. Comparando os resultados encontrados com os da referida autora, percebe-se que para o ensaio com $60 \%$ de $\mathrm{MO}$, a condutividade hidráulica encontrada foi menor. Para o ensaio com $40 \%$ de $\mathrm{MO}$, a condutividade encontrada por Triginelli (2015) apresentou um valor menor do que o encontrado neste trabalho, porém manteve-se na mesma ordem de grandeza, $10^{-5} \mathrm{~m} \mathrm{~s}^{-1}$. Uma possível explicação para essa diferença pode ser devido ao tipo de matéria orgânica utilizada em seu trabalho, pois foram utilizados apenas restos de comida (MO mais fluida) como matéria orgânica no ensaio. Já a MO utilizada no presente trabalho era mais diversificada (restos de comida, de frutas, galhos e folhas, entre outros).

\section{Teor de água}

Os valores de teor de água inicial e final, ou seja, correspondente à capacidade de campo, dos resíduos para os ensaios com peso específico de $5 \mathrm{kN}^{-3}$ são apresentados na Tabela 5, e para os ensaios com peso específico de $3 \mathrm{kN} \mathrm{m}^{-3}$ na Tabela 6. Os resultados apresentados foram obtidos a partir da média aritmética de três determinações.

Observa-se que o teor de água ou umidade gravimétrica média final, correspondente à capacidade de campo, foi superior à umidade gravimétrica média inicial em todos os ensaios. .

Tabela 5 - Valores de teor de água inicial e final, expresso em $\mathrm{kg}$, dos resíduos para ensaios com $5 \mathrm{kN} \mathrm{m}^{-3}$.

\begin{tabular}{cccccccc}
\hline & & \multicolumn{3}{c}{$40 \%$ de MO } & \multicolumn{3}{c}{$60 \%$ de MO } \\
\cline { 2 - 8 } Teor de água & Bandeja & 1 & 2 & 3 & 1 & 2 & 3 \\
inicial & MBU(g) & 3480 & 3461 & 3456 & 3469 & 3461 & 3457 \\
& MBS (g) & 2520 & 2434 & 2488 & 2317 & 2314 & 2318 \\
& W (\%) & 27,59 & 29,67 & 28,01 & 33,21 & 33,14 & 32,95 \\
\cline { 2 - 8 } & W médio $(\%)$ & & 28,42 & & & 33,10 & \\
\hline
\end{tabular}




\begin{tabular}{|c|c|c|c|c|c|c|c|}
\hline \multirow{6}{*}{ Teor de água final } & & \multicolumn{3}{|c|}{$40 \%$ de $\mathrm{MO}$} & \multicolumn{3}{|c|}{$60 \%$ de $\mathrm{MO}$} \\
\hline & Bandeja & 1 & 2 & 3 & 1 & 2 & 3 \\
\hline & $\operatorname{MBU}(\mathrm{g})$ & 3472 & 3470 & 3466 & 3474 & 3460 & 3459 \\
\hline & $\operatorname{MBS}(\mathrm{g})$ & 2239 & 2114 & 2126 & 2205 & 2066 & 2126 \\
\hline & W (\%) & 35,51 & 39,08 & 38,66 & 36,53 & 40,29 & 38,54 \\
\hline & $\mathrm{W}_{\text {médio }}(\%)$ & & 37,75 & & & 38,45 & \\
\hline
\end{tabular}

*MBU = massa bruta úmida; MBS = massa bruta seca; $\mathrm{W}(\%)=$ teor de água

Tabela 6 - Valores de teor de água inicial e final, expresso em $\mathrm{kg}$, dos resíduos para ensaios com $3 \mathrm{kN} \mathrm{m}^{-3}$.

\begin{tabular}{|c|c|c|c|c|c|c|c|c|c|c|}
\hline \multirow{6}{*}{$\begin{array}{l}\text { Teor de água } \\
\text { inicial }\end{array}$} & & \multicolumn{3}{|c|}{$20 \%$ de $\mathrm{MO}$} & \multicolumn{3}{|c|}{$40 \%$ de $\mathrm{MO}$} & \multicolumn{3}{|c|}{$60 \%$ de $\mathrm{MO}$} \\
\hline & Bandeja & 1 & 2 & 3 & 1 & 2 & 3 & 1 & 2 & 3 \\
\hline & $\operatorname{MBU}(\mathrm{g})$ & 3480 & 3455 & 3456 & 3474 & 3455 & 3462 & 3475 & 3456 & 3459 \\
\hline & $\operatorname{MBS}(\mathrm{g})$ & 2601 & 2642 & 2556 & 2519 & 2546 & 2528 & 2299 & 2293 & 2332 \\
\hline & W (\%) & 25,26 & 23,53 & 26,04 & 27,49 & 26,31 & 26,98 & 33,84 & 33,65 & 32,58 \\
\hline & $\mathrm{W}_{\text {médio }}(\%)$ & \multicolumn{3}{|c|}{24,94} & \multicolumn{3}{|c|}{26,93} & \multicolumn{3}{|c|}{33,36} \\
\hline \multirow{6}{*}{$\begin{array}{l}\text { Teor de água } \\
\text { final }\end{array}$} & & \multicolumn{3}{|c|}{$20 \%$ de $\mathrm{MO}$} & \multicolumn{3}{|c|}{$40 \%$ de $\mathrm{MO}$} & \multicolumn{3}{|c|}{$60 \%$ de $\mathrm{MO}$} \\
\hline & Bandeja & 1 & 2 & 3 & 1 & 2 & 3 & 1 & 2 & 3 \\
\hline & $\operatorname{MBU}(\mathrm{g})$ & 3472 & 3451 & 3452 & 3477 & 3456 & 3457 & 3479 & 3452 & 3456 \\
\hline & $\operatorname{MBS}(\mathrm{g})$ & 2154 & 2167 & 2167 & 2264 & 2244 & 2101 & 2104 & 2032 & 2062 \\
\hline & W (\%) & 37,96 & 37,21 & 37,22 & 34,89 & 35,07 & 39,22 & 39,52 & 41,14 & 40,34 \\
\hline & $\mathrm{W}_{\text {médio }}(\%)$ & & 37,46 & & & 36,39 & & & 40,33 & \\
\hline
\end{tabular}

*MBU = massa bruta úmida; MBS = massa bruta seca; $\mathrm{W}(\%)=$ teor de água

Verificou-se que quanto maior o teor de matéria orgânica maior é a umidade gravimétrica, o que corrobora com o trabalho de Landva e Clark (1990).

No entanto, percebe-se que nos ensaios com RSU compactados a $3 \mathrm{kN} \mathrm{m}^{-3}$, a umidade gravimétrica média final correspondente à capacidade de campo foi irrisoriamente inferior no ensaio de $40 \%$ de MO, quando comparado com o de $20 \%$ de MO. Tal fato pode ter ocorrido devido a maneira com que a amostra de $20 \%$ de MO foi retirada do permeâmetro para realização do ensaio de umidade, uma vez que durante o processo não há controle da quantidade de matéria orgânica presente na amostra, o que provavelmente ocasionou em um valor maior de umidade quando comparada com a amostra do ensaio com $40 \%$ de MO.

\section{Capacidade de campo}

$\mathrm{Na}$ Tabela 7 são apresentados os valores encontrados para a capacidade de campo dos resíduos para os ensaios com 3 e $5 \mathrm{kN} \mathrm{m}^{-3}$. 
Tabela 7 - Valores encontrados da capacidade de campo dos RSU.

\begin{tabular}{ccc}
\hline Peso específico & \% MO & $\boldsymbol{\theta}(\mathbf{\%})$ \\
\hline \multirow{2}{*}{$5 \mathrm{kN} \mathrm{m}^{-3}$} & 20 & - \\
& 40 & 14,98 \\
& 60 & 14,72 \\
\hline \multirow{2}{*}{$3 \mathrm{kN} \mathrm{m}^{-3}$} & 20 & 9,17 \\
& 40 & 8,77 \\
& 60 & 9,25 \\
\hline
\end{tabular}

Não foi obtido a capacidade de campo para o ensaio com $20 \%$ de $\mathrm{MO}$ e peso específico de $5 \mathrm{kN} \mathrm{m}^{-3}$, pelo fato de não ter sido possível determinar o teor de água inicial e final desta amostra.

Com base nos resultados apresentados na Tabela 7 , nota-se que os resíduos sólidos urbanos compactados com peso específico de $5 \mathrm{kN} \mathrm{m}^{-3}$ apresentaram uma maior capacidade de campo do que os resíduos compactados a $3 \mathrm{kN} \mathrm{m}^{-3}$, ou seja, foi verificado que quanto maior o peso específico, maior o valor da capacidade de campo, fato também constatado por Ribeiro et al (2013). Fazendo uma correlação com solos, possivelmente esse fato se deu pela massa dos resíduos mais compactada $(5 \mathrm{kN}$ $\mathrm{m}^{-3}$ ) apresentar uma maior quantidade de pequenos poros, sendo estes os responsáveis por reter a água. Já na massa dos resíduos compactados a $3 \mathrm{kN} \mathrm{m}^{-3}$, os resíduos se encontram mais soltos, apresentando uma grande quantidade de "macroporos", sendo estes incapazes de reter água proporcionando uma maior drenagem.
Ressalta-se que para o mesmo peso específico, a diferença do valor da capacidade de campo para cada porcentagem de MO foi muito pequena. Rocha (2008) observou em seu trabalho que resíduos mais novos, portanto com alta quantidade de matéria orgânica, apresentaram uma maior capacidade de campo, o que não pode ser verificado no presente trabalho.

\section{CONCLUSÕES}

O permeâmetro mostrou-se adequado para obtenção de valores de condutividade hidráulica e de capacidade de campo dos resíduos.

Há influência do peso específico dos RSU na determinação da condutividade hidráulica e capacidade de campo dos mesmos, pois observou-se que:

-Quanto maior o peso específico, menor é a condutividade hidráulica, devido à diminuição dos espaços vazios por onde o líquido percola. 
-Quanto maior o peso específico, maior é a capacidade de campo, devido à maior presença de microporos responsáveis por reter a água.

\section{REFERÊNCIAS}

BARROS, H. L. Estudo de balanço hídrico em aterro sanitário por meio de lisímetros de grandes dimensões. 2004. 112 p. Dissertação (Mestrado em Engenharia Civil), Universidade Federal de Viçosa, Viçosa, 2004.

BORGATTO, A.V.A. (2010). Estudo das propriedades geomecânicas de resíduos sólidos urbanos pré-tratados. 2010. 271

f. Tese (Doutorado em Engenharia Civil),Universidade Federal do Rio de Janeiro, Rio de Janeiro.

BORGATTO, A.V.A. Estudo do efeito fibra e da morfologia na estabilidade de aterros de resíduos sólidos urbanos. 2006. 157 p. Dissertação (Mestrado em Ciências em Engenharia Civil),Universidade Federal do Rio de Janeiro, Rio de Janeiro, 2006.

BOSCOV, M. E. G., ABREU, R. C. de. (2000). Aterros Sanitários. In: Previsão de desempenho - comportamento real aterros sanitários, taludes, túneis, fundações, aterros sobre solos moles, $\mathrm{pp}$. 7-44, São Paulo: ABMS/NRSP.

BRASIL. Lei $\mathbf{n}^{\mathbf{0}} \mathbf{1 2 . 3 0 5}$, de 2 de agosto de 2010. Institui a Política Nacional de Resíduos Sólidos; altera a Lei n ${ }^{\mathbf{0}}$ 9.605, de 12 de fevereiro de 1998; e dá outras providências. Disponível em:

$<$ http://www.planalto.gov.br/ccivil_03/_at o2007-2010/2010/lei/112305.htm>. Acesso em: 03 de abril de 2016.

CARVALHO, A.R. (2002). Percâmetro: um novo equipamento para medidas de parâmetros geotécnicos de resíduos sólidos. Desenvolvimento e aplicação no aterro sanitário de Santo André - São Paulo. 2002. 120 p. Dissertação (Mestrado em Engenharia Civil), Universidade Federal de Viçosa, Viçosa.

CARVALHO, A.R. (2006).

Desenvolvimento de um equipamento para determinação de parâmetros geotécnicos de resíduos sólidos. 2006. 160 f. Tese (Doutorado em Ciências em Engenharia Civil). Universidade Federal do Rio de Janeiro, Rio de Janeiro.

\section{CAlle, J. A. C. Comportamento} geomecânico de resíduos sólidos urbanos. 2007. 160 f. Tese (Doutorado em Ciências em Engenharia Civil), Universidade Federal do Rio de Janeiro, Rio de Janeiro, 2007.

DIXON, N., JONES, D. R. V. (2005) Engineering properties of municipal solid waste. Geotextiles and geomembranes. vol. $25, \mathrm{n}^{\mathrm{o}} .3$, pp. 205-212.

DURMUSOGLU, E., SANCHEZ, I. M., CORAPCIOGLU, M. Y. (2006).

Permeability and compression characteristics of municipal solid waste samples. Journal Environmental Geology. vol. 50, nº 6, pp. 773-786.

EL-FADEL, M.; BOU-ZEID, E.; CHAHINE, W.; ALAYLI, B. Temporal variation of leachate quality from presorted and baled municipal solid waste with high organic and moisture content. Waste Management, v.22, n.3, p.269-282, 2002.

FRANCO, C. S. (2012). Caracterização gravimétrica dos resíduos sólidos domiciliares e percepção dos hábitos de descarte no sul de Minas Gerais. 2012, 142 p. Dissertação (Mestrado em Engenharia Agrícola), Universidade Federal de Lavras, Lavras. 
LANDVA, A. O.; CLARK, J. I. (1990). Geotechnics of Waste Fills - Theory and Practice. ASTM STP n ${ }^{\circ} 1070$, Arvid Landva, G. David Knowles, editors, American Society for Testing and Materials, Philadelphia, p.86-103.

LEMARE NETO, A. L. (2004).

Resistência ao cisalhamento de resíduos sólidos urbanos e de materiais granulares com fibras. 2004. 190 f. Tese (Doutorado em Ciências em Engenharia Civil), Universidade Federal do Rio de Janeiro, Rio de Janeiro.

LINS, E. A. M. A utilização da capacidade de campo na estimativa do percolado gerado no aterro da Muribeca. 2003. 125 f. Dissertação, Universidade Federal de Pernambuco

MACHADO, S. L.; FARD-KARIMPOUR, M.; SHARIATMADARI, N.;

CARVALHO, M. F.; NASCIMENTO, J.

F. Evaluation of the geotechnical properties of MSW in two Brazilian landfills. Waste Management, v. 30, n. 12, p. 2579-2591, 2010.

MORTATTI, B. C. (2013). Determinação da condutividade hidráulica e análise química do lixiviado de resíduos sólidos urbanos utilizando permeâmetros de grandes dimensões. 2013. $110 \mathrm{p}$.

Dissertação (Mestrado em Geociências), Universidade Estadual de Campinas, Campinas.

RIBEIRO, A. G. C.; AZEVEDO, R. F.; ROCHA, E. F.; AZEVEDO, I. C. D.; LORETO, A. S.. Determinação de parâmetros mecânicos de resíduos sólidos urbanos por meio de ensaios realizados em laboratório. Geotecnia, n. 127, p. 81-104, 2013.

ROCHA, E. F. (2008). Determinação da condutividade hidráulica e da capacidade de campo de resíduos sólidos urbanos. 2008. 88 p. Dissertação (Mestrado em Engenharia Civil), Universidade Federal de Viçosa, Viçosa.

SILVEIRA, A. M. M. Estudo do peso específico de resíduos sólidos urbanos. 2004. 101 p. Dissertação (Mestrado em Ciências em Engenharia Civil), Universidade Federal do Rio de Janeiro, Rio de Janeiro, 2004

TRIGINELLI, B.P. (2015). Influência da composição gravimétrica na condutividade hidráulica de resíduos sólidos urbanos. Lavras: UFLA, 2015. 48 p.

YUEN S. T. S., WANG Q. J., STYLES J. R., MCMAHON T. A. 2001. Water balance comparison between a dry and a wet landfill: a full-scale experiment. Journal of Hydrology, 251: 29-48. 